\title{
MODELO MATEMÁTICO PARA MINIMIZAR LOS COSTOS DE PRODUCCIÓN EN UNA ENSAMBLADORA DE VEHÍCULOS - ÁREA DE ENSAMBLAJE.
}

\section{Mathematical model to minimize production costs in a vehicle assembler - assembly area.}

\author{
${ }^{1}$ José Gavidia* (iD) ${ }^{2}$ Antonio Meneses (iD), ${ }^{3}$ Lourdes Zúñiga (D), ${ }^{1}$ Christian Mariño iD.
}

${ }^{1}$ Universidad Técnica de Ambato, Facultad de Sistemas, Electrónica e Industrial, Carrera de Ingeniería Industrial, Ambato, Ecuador.

${ }^{2}$ Universidad Nacional de Chimborazo, Facultad de Ingeniería, Carrera de Ingeniería Industrial, Riobamba, Ecuador.

${ }^{3}$ Escuela Superior Politécnica de Chimborazo, Facultad de Informática y Electrónica, Carrera Ingeniería en Telecomunicaciones, Riobamba, Ecuador.

*jl.gavidia@uta.edu.ec

$\mathrm{R}$ esumen

El trabajo de investigación tiene como objetivo diseñar un modelo matemático para minimizar los costos de producción, mediante la aplicación del software Lingo 19 en el ensamble del vehículo Modelo HAVAL M4 AC 1.5 5P 4X2 producido por la empresa Ciauto Cía. Ltda. Se diseñó un instrumento (Check List) para el levantamiento de la información. En el análisis de la Capacidad Instalada (CI) se obtuvo un valor de doce (12) vehículos/diarios, donde el $50 \%$ se ensambla el modelo más vendido por la empresa, es decir, seis (6) vehículos Modelos M4 por día, con un costo de producción total al año de USD 198.052,50. En base a los datos del trabajo de investigación se concluye, dotar de una herramienta matemática que minimice los costos de producción en el área de ensamble de la empresa Ciato Cía. Ltda. de forma eficiente y sostenible a corto, mediano y largo plazo, con el fin de generar una gestión eficiente de los recursos. Se recomienda aplicar el Modelo Matemático de Programación Lineal para minimizar los costos de producción para cualquier área de en una ensambladora de vehículos, como suelda, pintura o desempaque.

Palabras clave: Capacidad, Ensamble, Modelo, Planeación, Producción, Desempaque.

\section{A bstract}

The objective of the research work is to design a mathematical model to minimize production costs, through the application of the Lingo 19 software in the assembly of the HAVAL M4 AC 1.5 5P 4x2 vehicule model produced by the company Ciauto Cía. Ltda. An instrument (Check List) was designed to collect the information. In the analysis of the Installed Capacity (IC), a value of twelve (12) vehicles / day was obtained, where $50 \%$ of the most sold model is assembled by the company, that is, six (6) M4 Models vehicles per day, with a total production cost per year of USD 198,052.50. Based on the data from the research work, it is concluded to provide a mathematical tool that minimizes production costs in the assembly area of the company Ciauto Cía. Ltda in an efficient and sustainable way in the short, medium, and long term, to generate an efficient management of resources. It is recommended to apply the Linear Programming Mathematical Model to minimize production costs for any area of a vehicle assembler, such as welding, painting or unpacking.

Keywords: Capacity, Assembly, Model, Planning, Production, Unpacking.

Fecha de recepción: 17-06-2021 Fecha de aceptación: 03-09-2021 Fecha de publicación: 13-10-2021

\section{INTRODUCCIÓN}

El objetivo del trabajo de investigación se enfocó en Diseñar un Modelo Matemático para mini- mizar los costos de producción, que permita la gestión eficiente de los recursos en una empresa ensambladora de vehículos. La metodología se fundamentó en un enfoque de investigación 
cuantitativo, con un método inductivo y deductivo, haciendo uso de una investigación bibliográfica y documental (1-3).

El proceso de llegar a plantear el modelo matemático para minimizar los costos de producción, que permita la gestión eficiente de los recursos en una empresa ensambladora de vehículos, se basa en la aplicación del software matemático Lingo 19 , con el cual se logra minimizar la función objetivo, teniendo en cuenta las restricciones, donde con la aplicación de este software se obtiene los costos de cinco (5) aspectos más influyentes inmersos en la producción. (costo de un trabajador por mes, costo de contratar y capacitar un trabajador, costo de despedir un trabajador, costo de mantener inventario, costo de inventario agotado) (4).

Revisando el estado del arte podemos encontrar investigaciones similares, como el caso de un "Modelo matemático para la planificación de la producción del sector cuero en la parroquia de Quisapincha" Ambato Ecuador, donde el estudio fue de elaborar un modelo matemático que se adecue a las necesidades de mejora económica de este sector, con el fin de planificar adecuadamente la producción mediante el uso de una herramienta cuantitativa que ayude a fundamentar la toma de decisiones y mejorar la gestión administrativa, como resultado lograron maximizar sus utilidades, como podemos observar, las ventajas de implementar estos modelos es minimizar costos y maximizar ganancias $(5,6)$.

En el desarrollo del presente trabajo de investigación, se emplea un instrumento para el levantamiento de la información (Check List) y el software matemático Lingo 19, con ello se diseña el modelo matemático que permite minimizar los costos de producción, optimizando los recursos inmersos en esta actividad (7).

\section{MATERIALES Y MÉTODOS}

- Materiales.

Para el desarrollo del presente trabajo de investigación se hace uso de los siguientes materiales: - $\quad$ Plan de producción vigente.

\section{- $\quad$ Check List \\ - Software Matemático Lingo versión 19 \\ - Computador y equipos de oficina.}

\section{- Metodología.}

La investigación empieza con el estudio del estado del arte, y con el análisis documental del proceso de producción actual de la planta, haciendo uso de un Check List, el cual se aplica en el área de ensamble para el modelo M4, el mismo que es el más significativo para la empresa Ciauto Cia. Ltda. Donde se determina reducir los costos de producción enmarcados en un año, para ello se selecciona cinco (5) aspectos más representativos en esta actividad (costo de un trabajador, costo de contratar y capacitar a un trabajador, costo de despedir a un trabajador, costo de mantener inventario y costo de inventario agotado). Para diseñar el modelo matemático que minimice estos costos de producción, se hace uso del software matemático Lingo $19(8,9)$.

\section{Área de Estudio}

La determinación del modelo matemático para minimizar los costos de la producción se lo hace en el área de ensamble de vehículos compactos, conformada por diez estaciones de trabajo denominadas: E1, E2, E3, E4, E5, E6, E7, E8, E9, E10, distribuidas en las tres líneas de ensamble que la constituyen siendo estas: Línea TRIM, Línea Chasis, Línea Liberación o final (10).

\section{Planificación de la Producción}

Se refiere esencialmente a la cantidad de fabricación de artículos y vigilar que se haga como se planeó, es decir, el control se refiere a la verificación para que se cumpla con lo planeado, reduciendo a un mínimo las diferencias del plan original (11).

\section{Producto Ensamblado}

En la Figura 1, se ilustra el Vehículo HAVAL M4 AC 1.5 5P 4x2 TM resultante de la línea de ensamble. El proceso se desarrolla a medida que el auto pasa por las diferentes estaciones de trabajo (E1 a E10) dentro del área de ensamblaje, poste- 
riormente pasa por las estaciones de pruebas de calidad, hasta obtener el producto final que será dispuesto en el mercado nacional.

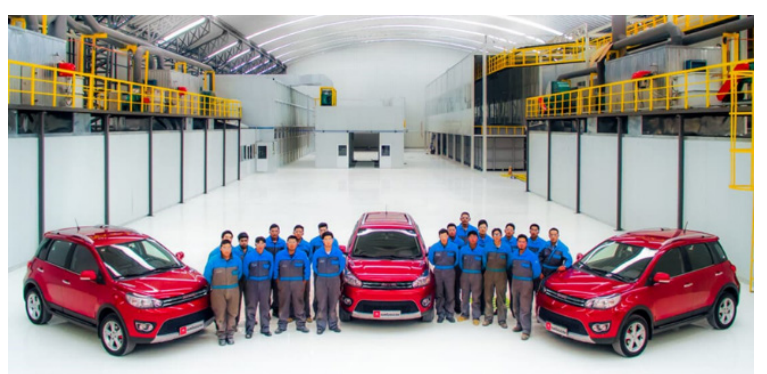

Figura 1. Vehículo Great Wall M4, 1.5 5P 4X2 TM.

\section{Resumen del Diagrama de Proceso de ensamble - Modelo M4}

En las instalaciones del área de producción de vehículos compactos de la empresa CIAUTO se ejecutan diversas actividades para obtener el Modelo M4 totalmente armado, para ello, a continuación, se presenta en resumen el diagrama de proceso de ensamblaje del modelo M4, (tabla 1), tomando en cuenta que el proceso de ensamble demanda la utilización de herramientas e instrumentos de trabajo calibrados para realizar las operaciones adecuadamente $(12,13)$.

\begin{tabular}{|l|c|c|c|c|c|c|}
\hline \multicolumn{7}{|c|}{ RESUMEN PROCESO } \\
\hline \multicolumn{1}{|c|}{ Actividad } & Cantidad & Tiempo (min) & Distancia (m) & Ahorro (\$) & Observaciones \\
\hline Operación & & 30 & $19: 50: 33$ & $\ldots \ldots \ldots$ & & \\
\hline Transporte & $\Rightarrow$ & 13 & $0: 38: 28$ & 37 & & \\
\hline Inspección & $\square$ & 12 & $1: 20: 41$ & $\ldots \ldots \ldots$ & & \\
\hline Demora & $\mathbf{D}$ & 4 & $0: 03: 57$ & $\ldots \ldots \ldots$ & & \\
\hline Almacenaje & $\nabla$ & 0 & 0 & $\ldots \ldots \ldots$ & & \\
\hline \multicolumn{2}{c}{ Total } & & $\mathbf{2 1 : 5 3 : 3 4}$ & 37 & & \\
\hline
\end{tabular}

Tabla 1. Matriz resumen del Diagrama de Proceso de ensamble - Modelo M4.

Determinación de tiempos de las actividades que se ejecutan en cada estación de trabajo.

Para la obtención de los tiempos cronometrados de cada área de trabajo a lo largo de la línea de ensamble en la Ensambladora de vehículos Ciauto Cía. Ltda., se considera en primera instancia la jornada laboral que se aplica en la organización, para lo cual el personal se acoge a una jornada de trabajo que comprende un horario de 7am a $4 \mathrm{pm}$, detallada en la Tabla 2, de esta se tiene que el tiempo disponible de ensamblaje es de 7 horas con 40 minutos correspondiente a 460 minutos $(14,15)$.

\begin{tabular}{ccc} 
No & Actividad & Hora \\
\hline 1 & Ingreso & $07: 00$ \\
2 & Periodo de Reunión diaria con el LET & $07: 10$ \\
\hline 3 & Inicio del Breack (periodo de descanso) & $09: 45$ \\
\hline 4 & Regreso a los puestos de trabajo & $10: 00$ \\
\hline 5 & Inicio del periodo de almuerzo & $12: 45$ \\
\hline 6 & Ingreso a los puestos de trabajo & $13: 30$ \\
\hline 7 & Fin de jornada & $15: 50$ \\
\hline 8 & Limpieza de estaciones y entrega de & $16: 00$ \\
& herramientas & $16: 00$
\end{tabular}

Tabla 2. Jornada Laboral CIAUTO.

Balance de las líneas de producción en el área de ensamble del Modelo M4

Con el fin de equilibrar las cargas de trabajo en las estaciones del área de ensamble de la Ensambladora de vehículos Ciauto Cía. Ltda., se realiza el balance de las líneas productivas desarrollando en primera instancia el cálculo del takt time y el número de estaciones, para mediante esto realizar el diagrama de equilibrio de la línea de ensamble.

Mediante el método de balanceo de líneas se agrupa las operaciones consecutivamente de tal forma que los operarios tengan una misma cantidad de carga de trabajo con el fin de aprovechar al máximo la mano de obra, la utilización del equipo de manera que el tiempo inactivo de todas las estaciones de trabajo sea el menor posible en la producción del modelo M4, para ello se analiza la jornada laboral de la organización como base fundamental del cálculo del takt time con el propósito de que todas las estaciones se ajusten al plan de producción establecido de doce (12) vehículos diarios $(2,16)$.

\section{Cálculo del Takt Time}

Del análisis precedente realizado de la jornada laboral de la Ensambladora de vehículos Ciauto Cía. Ltda., detallada en la Tabla 2, se tiene que el tiempo disponible es de 7 horas con 40 minutos correspondiente a 460 minutos utilizables por el operario de cada subestación para cumplir el ensamble de 12 unidades, con estos datos calculamos el Takt Time, aplicando la Ec. 1 (17).

$$
\text { Takt Time }(T T)=\frac{\text { Tiempo Disponible de Trabajo }}{\text { Demanda de Producción }}
$$




$$
\begin{gathered}
\text { Takt Time }(\boldsymbol{T T})=\frac{460 \mathrm{~min}}{12 \text { unidades }} \\
\text { Takt Time }(\boldsymbol{T T})=38,33 \frac{\mathrm{min}}{\text { unidad }} \\
\text { Takt Time }(\boldsymbol{T} T)=38: 33 \approx 39 \frac{\mathrm{min}}{\text { unidad }}
\end{gathered}
$$

El Takt Time calculado indica que el ritmo de producción de los automóviles modelo M4 demandado por el cliente debe finalizar en una duración de 38 minutos con 19.8 segundos, aproximadamente 39 minutos, es decir, en este ciclo de tiempo debe salir un auto completamente ensamblado (18).

Análisis de la capacidad instalada en el área de ensamble de CIAUTO.

Con el fin de conocer el volumen de producción que la Ensambladora de vehículos Ciauto Cía. Ltda., podría generar en base a su infraestructura, adecuación de sus instalaciones y de acuerdo con las máquinas-herramientas con la que cuenta cada estación de trabajo, se realizan diferentes cálculos que ayuden a la empresa a identificar su producción máxima, generando una estrategia competitiva acorde a la demanda del mercado (19).

Cálculo del Índice de Productividad (IP)

Para la obtención del IP se emplea la ecuación (2), ocupando los datos presentes en la Tabla 3

\begin{tabular}{ccc}
\hline No & Actividad & Hora \\
\hline 1 & T Laboral (min) & 480 \\
\hline 2 & T Reuniones (min) & 20 \\
\hline 3 & T Disponible (min) & 460 \\
\hline 4 & Unidades Planificadas (u) & 12 \\
\hline 5 & Takt time (minutos) & 38 \\
\hline 6 & Suplementos & 1,11 \\
\hline 7 & TT con suplementos(min) & $0: 46: 37$
\end{tabular}

Tabla 3. Datos para la planificación de la producción.

$$
\begin{gathered}
I P=\frac{\text { Producción diaria }}{\text { Tiempo disponible }}(2) \\
I P=\frac{12 \text { vehiculos }}{460 \text { minutos }} \\
I P=0.026 \frac{\text { vehiculos }}{\text { minutos }} * \frac{60 \text { minutos }}{1 h} \\
I P=\mathbf{1 . 5 6} \frac{\text { vehiculos }}{\text { hora }}
\end{gathered}
$$

Del cálculo se obtiene como resultado que en cada estación de trabajo de la Línea de ensamble de vehículos compactos se debe producir un vehículo completamente armado y un avance del $56 \%$ en el ensamblaje del siguiente vehículo en el transcurso de cada hora de la jornada laboral.

\section{Cálculo de la Productividad}

Para proceder a realizar el respectivo análisis de productividad se toma el valor del número de operarios reales $(\mathrm{NOR}=30)$ que están presentes en el área de ensamble del Modelo M4, esto con el fin de establecer un dato verídico con respecto al aprovechamiento de los recursos existentes en las instalaciones de la organización. Se emplea la ecuación (3) mediante la cual se procede hacer el cálculo pertinente.

Sabemos que: 30 operarios producen 12 unidades por día.

$$
\begin{gathered}
\text { Productividad }=\frac{\text { Producción diaria }}{\text { Horas hombre empleadas }} \\
\text { Productividad }=\frac{12 \text { vehículos }}{8 h} \\
\text { Productividad }=1.5 \frac{\text { vehículos }}{h}
\end{gathered}
$$

Si 1.5 Unidades por hora producen 30 operarios, ¿Cuánto producirá un operario?

$$
\begin{gathered}
\text { Productividad }=\frac{(1.5 \text { vehiculos } / \text { hora }) * 1 \text { operario }}{30 \text { operarios }} \\
\text { Productividad }=0.05 \frac{\text { vehiculos }}{\text { hora }} * \text { operario }
\end{gathered}
$$

Del cálculo realizado se establece que la productividad por hora se tiene un valor de 0,05 , es decir, que un operario realiza aproximadamente un $5 \%$ del ensamble de un auto por cada hora de la jornada laboral.

\section{Cálculo de la Capacidad Instalada (CI)}

Con los cálculos previos realizados se procede a efectuar el respectivo análisis de la Capacidad Instalada, para ello se emplea la ecuación 4.

$$
\begin{gathered}
C I=\frac{\text { vehículos }}{\min } * \text { min diarios trabajdos } * \text { dias trabajados } \\
C I=0.026 \frac{\text { vehículos }}{\min } * 460 \text { min } * 5 \text { dias } \\
C I=59,8 \frac{\text { vehículos }}{\text { semanales }} \\
C I=59,8 \frac{\text { vehículos }}{\text { semanales }} * \frac{1 \text { semana }}{5 \text { días }} \\
C I=11.96 \frac{\text { vehículos }}{\text { diarios }}
\end{gathered}
$$

Del cálculo realizado se obtiene que la Capacidad Instalada en la Línea de ensamble de vehículos 
compactos es de 59,8 vehículos/semanales, esto significa que la ensambladora en base a sus recursos, equipamiento e infraestructura está en la capacidad de ensamblar el 50\% de un Modelos M4 semanalmente. Del cálculo desarrollado para conocer la CI diaria se obtiene como resultado $11,96 \approx 12$ vehículos/diarios, de los cuales el $50 \%$ se ensambla el modelo más vendido por la Marca, (6 Modelos M4) este valor ratifica que el plan de producción fijado por el coordinador de ensamble para el Modelo M4 está bien dispuesto.

\section{Modelo Matemático de Programación Lineal}

Existen varias alternativas de solución para encontrar el plan de producción ideal, pues ahora se empieza a trabajar con el método matemático de Hansmann, F. y Hess, ya que es el modelo que más se acopla con la realidad del proceso de ensamble de la empresa, en donde se comienza definiendo cada uno de los parámetros o variables que intervienen en este proceso, los cuales se aprecian en la tabla $4(20,21)$.

\begin{tabular}{|c|c|c|}
\hline No. & Variable & Interpretación \\
\hline 1 & $t=$ & Indica el número de periodos, $t 1,2, \ldots, T$. \\
\hline 2 & $C_{t}^{W}=$ & Costo de un trabajador en el periodo $t$. \\
\hline 3 & $C_{t}^{C}=$ & Costo de contratar un trabajador en el periodo $t$ \\
\hline 4 & $C_{t}^{D}=$ & Costo de despedir a un trabajador en el periodo $t$ \\
\hline 5 & $C_{t}^{I}=$ & Costo de mantener una unidad en inventario en el periodo $t$ \\
\hline 6 & $C_{t}^{F}=$ & Costo del faltante de una unidad en el periodo t. \\
\hline 7 & $P_{t}=$ & Número de unidades producidas en el periodo t \\
\hline 8 & $W_{t}=$ & Número de trabajadores en el periodo t. \\
\hline 9 & $C_{t}=$ & Número de trabajadores contratados en el periodo t. \\
\hline 10 & $D_{t}=$ & Pronóstico de la demanda en el periodot \\
\hline 11 & $I_{t}=$ & Número de unidades en inventario en el periodo $t$ \\
\hline 12 & $F_{t}=$ & Número de unidades faltantes en el periodo $t$ \\
\hline 13 & $n_{t}=$ & Número de unidades que realiza un trabajador en el periodo \\
\hline
\end{tabular}

Tabla 4. Interpretación de Variables.

El objetivo de este modelo es minimizar el costo de cinco (5) recursos más influyentes que intervienen en la producción, para lo cual se procede armar la siguiente función objetivo (ecuación 5), para minimizar el costo de la producción anual en la Ensambladora de vehículos Ciauto Cía. Ltda. $(21,22)$.

minimizar $=\sum_{\mathrm{t}=1}^{T} C_{t}^{W} * P_{t}+C_{t}^{C} * C_{\mathrm{t}}+C_{\mathrm{t}}^{D} * D_{\mathrm{t}}+C_{\mathrm{t}}^{I} * I_{\mathrm{t}}+C_{t}^{F} * F_{\mathrm{t}}$

A continuación, se procede armar cada una de las restricciones vitales para el modelo de pro- gramación lineal, ya que dichas restricciones son de gran ayuda para la respectiva solución del problema.

- Se calcula el número de unidades que se puede producir en cada periodo, y esto con la ayuda de multiplicar el número de unidades que una persona puede realizar en un mes, por la variable del número de trabajadores disponibles en el mes. (Ecuación 6)

$$
P_{t} \leq n_{t}{ }^{*} W_{t} ; \quad 1 \leq t \leq T
$$

Se calcula el número de trabajadores necesarios en cada periodo, y esto mediante la suma de los trabajadores al inicio del año, más el número de trabajadores contratados, menos el número de trabajadores despedidos en el mes, lo que se detalla a continuación, para esto aplicamos la Ecuación 7.

$$
W_{t}=W_{t-1}+C_{t}-D_{t} ; \quad 1 \leq t \leq T
$$

- $\quad$ Finalmente, se encuentra el dato del número de inventario que va a existir en cada mes del año 2019, y esto a través de la operación algebraica del número de inventario en existencia, menos el número de inventario faltante, más la producción en cada mes y menos la demanda de unidades estipuladas para el año 2020. Para ello aplicamos la Ecuación 8.

$$
I_{t}-F_{t}=I_{t-1}-F_{t-1}+P_{t}-D_{\dot{t}} ; \quad 1 \leq t \leq T
$$

Donde $I_{t-1} \mathrm{y}, F_{t-1}$ son inventario inicial y ordenes atrasadas respectivamente, además cabe mencionar que todas estas variables se deben mostrar en forma entera positiva.

\section{Plan agregado en ensamble}

El proceso para obtener el Modelo M4 totalmente armado, se establece el tipo de producción apropiada para los 12 meses del año 2020 que optimice todos sus recursos y genere menos costos. Para una demanda con variación estacional, se planifica trabajar inicialmente con 30 operarios, además se determina los costos de producción para el plan agregado, los que se muestran en la Tabla 7. (23).

La función objetivo para minimizar los costos de producción para el Modelo M4, se la plantea a través de la ecuación cinco (5), utilizando para 
las constantes la información detallada en la Tabla 8. Una vez planteada la función objetivo, se procede a diseñar cada una de las restricciones, armadas dentro del programa Lingo 18. En la Figura dos (2), se presenta la codificación del modelo matemático, donde primero se muestra la declaración de las variables de tipo vectorial y matricial, posterior a ello se les asigna valores a las constantes del modelo y luego se plantea, la función objetivo, las restricciones para conocer la producción, el número de trabajadores y por último el número del inventario total para cada mes (20).

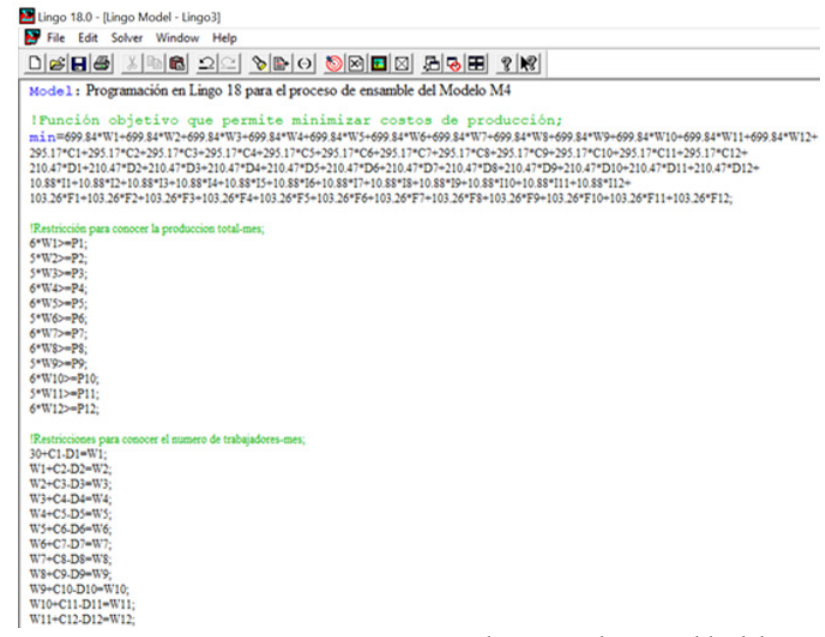

Figura 2. Programación en Lingo 18 para el proceso de ensamble del Modelo M4
Para encontrar el número de unidades que un trabajador puede realizar en un día, se emplea el tiempo estándar del proceso, para ello se realiza la siguiente regla de tres, tal como se la calculó anteriormente en los planes de producción tradicionales para el proceso de ensamble del Modelo M4, como se muestra a continuación.

30 operarios durante 0.641 horas ensamblan 1 unidad del Modelo M4. ¿Cuántas unidades del Modelo M4 ensamblará un operario en 7 h 40 $\min =7,67 \mathrm{~h}$ ?

$$
\begin{array}{ccc}
\text { Operarios } & \text { Horas } & \text { Ensamble M4 } \\
30 & 0.641 & 1 \\
1 & 7.67 & \mathrm{X} \\
\frac{(30)(0.641)}{1}=\frac{(1)(7.67)}{x} \Rightarrow x=\frac{(1)(7.67)}{(30)(0.641)} \Rightarrow x \approx 0.40 \frac{M 4}{\text { di } a}
\end{array}
$$

Luego calculamos los días disponible por mes, que resulta del producto días hábiles (tabla 5) por $50 \%$, este número multiplicamos por el número de unidades del Modelo M4 que realiza un operario en un día, y se obtiene el total de unidades que un trabajador puede producir en un mes, lo que se muestra en la tabla 6 .

En la tabla 5 se muestra la Demanda y los días hábiles por mes durante el año 2020.

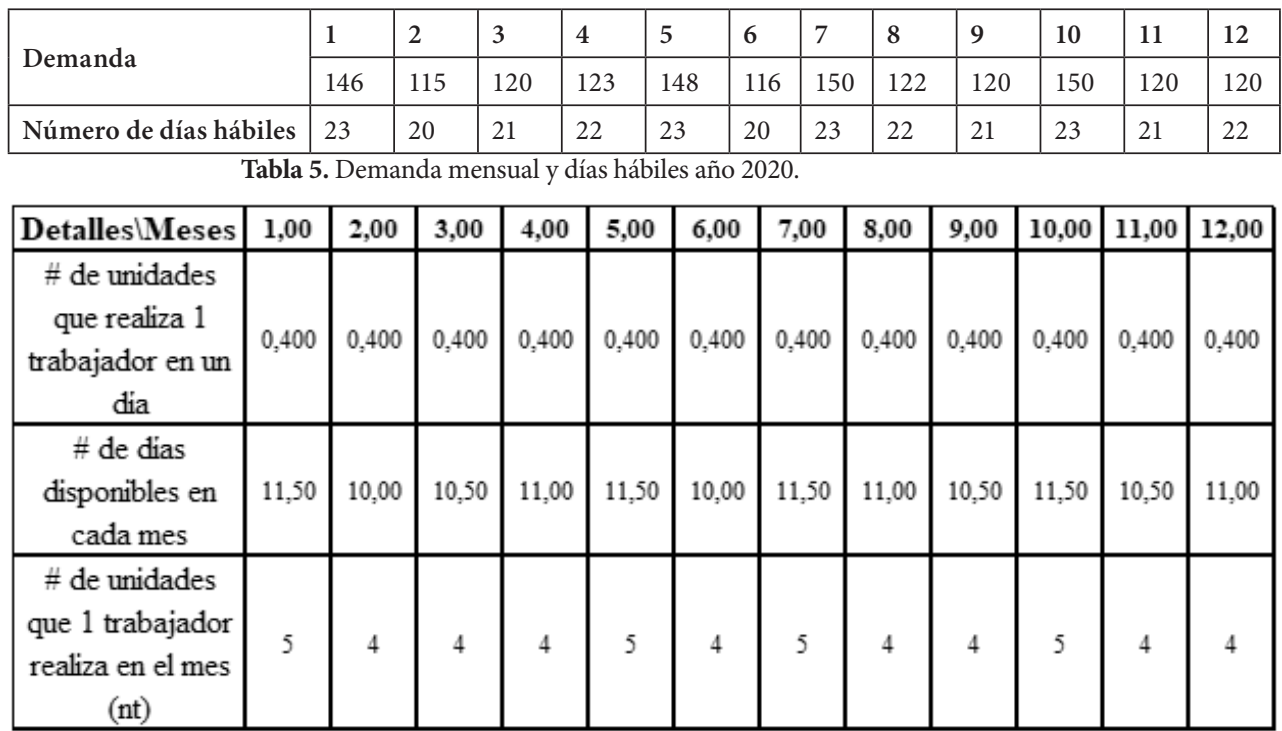

Tabla 6. Número de unidades que ensambla un trabajador al mes, del Modelo M4.

\section{RESULTADOS}

- Resolución con el método de programación lineal para el proceso de ensamble del Modelo M4

Una vez que se conoce cada uno de los datos que forman parte de la producción con sus respectivas variables, se comienza a armar el modelo de programación lineal dentro del programa Lingo 19 (23).

1. Cálculo de producción en cada mes. 


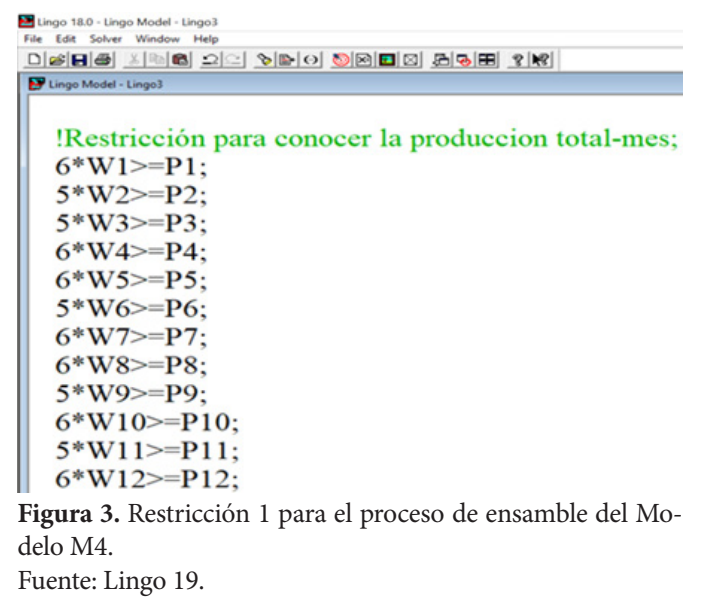

2. Cálculo de trabajadores en cada mes.

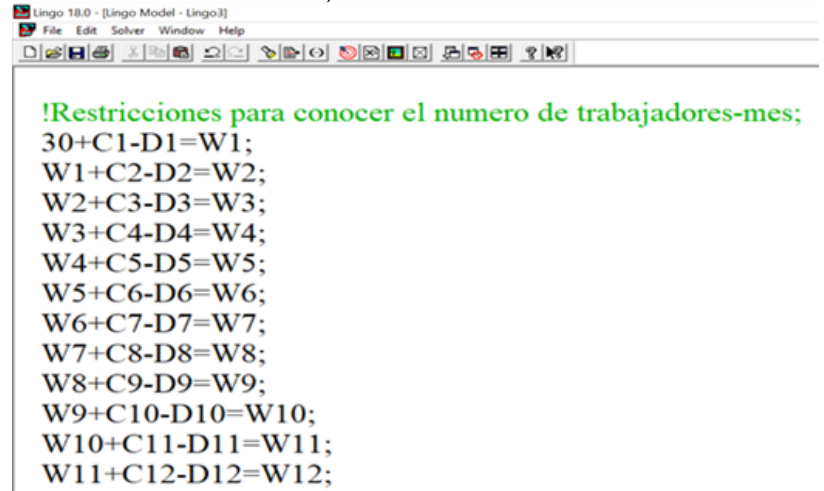

Figura 4. Restricción 2, No. de trabajadores para el proceso de ensamble Modelo M4

Fuente: Lingo 19.

3. Cálculo de inventario en cada mes.

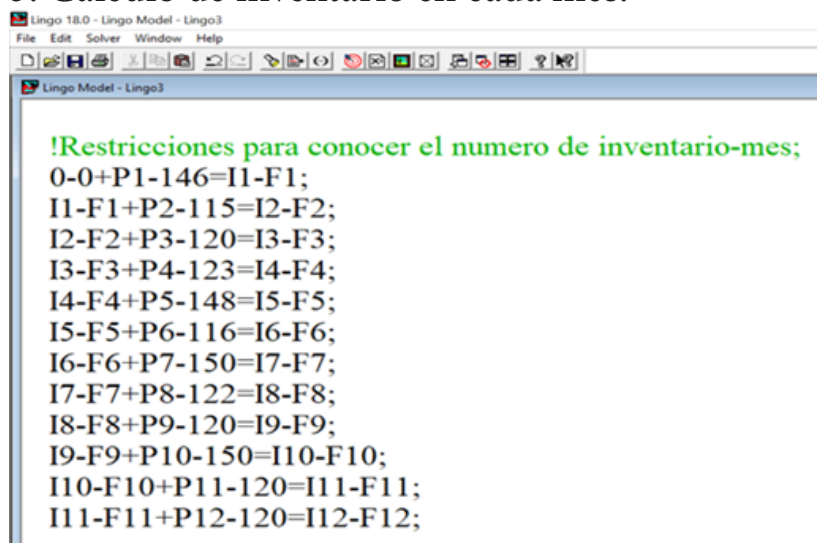

Figura 5. Restricción 3 inventario para el proceso de ensamble del Modelo M4.

Fuente: Lingo 19.

\section{Programar las variables a datos enteros.}

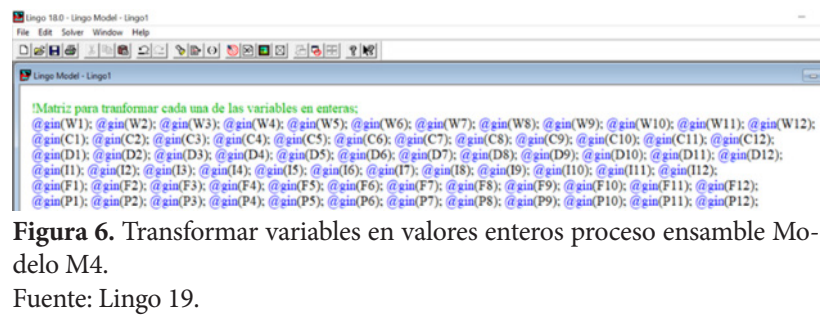

Resultados con el Método de Programación Lineal para el proceso de ensamble del Modelo M4.

区 Lingo 18.0 - [Solution Report - Lingo3]

File Edin Solver Window Help

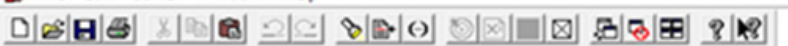

Global optimal solution found.
Objective value:
Objective bound:
Infeasibilities:
Extended solver steps:
Total solver iterations:
Elapsed runtime seconds:
Model class:
Total variables:
Nonlinear variables:
Integer variables:
Total constraints:
Nonlinear constraints:
Total nonzeros:
Nonlinear nonzeros:

Figura 7. Respuesta de Lingo 19 para el proceso de ensamble del Modelo M4.

En la figura 7, se muestra la solución de la función objetivo, Costo total de Producción al año, que es de \$198.052,50; además se detalla el número de pasos que se aplica para dar la solución que es de setecientos sesenta y seis (766) pasos, setenta y dos (72) variables y seis mil cuarenta y dos (6.042) iteraciones.

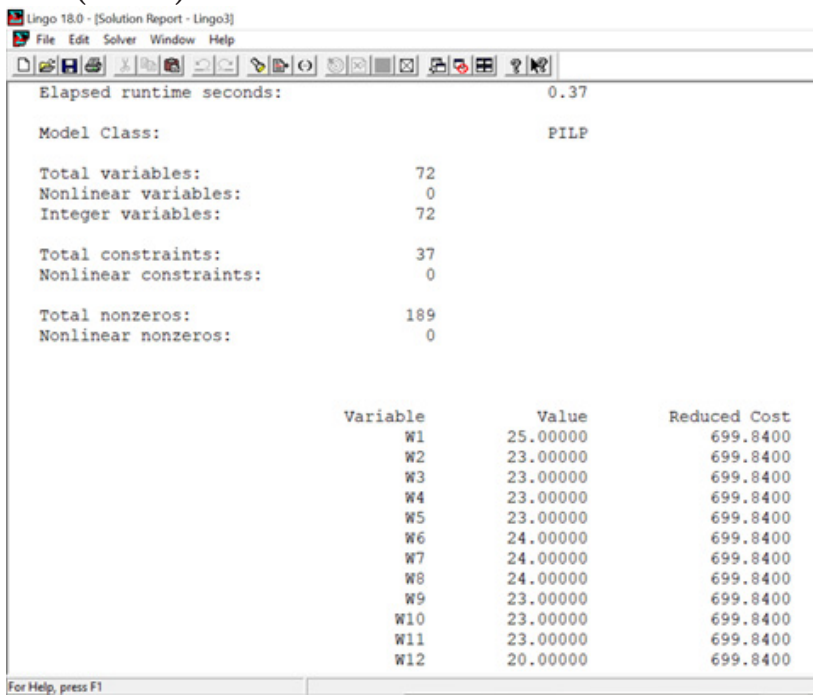

Figura 8. Cálculo del número de operarios por periodo.

La figura 8 , indica el número de operarios que deben existir en cada mes, expresado en la columna de Value, y el valor que debemos reducir para modificar la columna Value, expresada en la columna Reduced Cost. Que es el costo de un trabajador por mes, igual a, \$699.84.

La figura 9, enseña el número de operarios contratados en cada periodo, expresado en la columna Value, y los costos a reducir en la columna Reduced Cost. Que es el costo de contratar y capacitar un trabajador por mes, igual a, \$ 295.17 


\begin{tabular}{lll}
\hline $\mathbf{Q}] \mathbf{B}|\mathbf{B}| \boldsymbol{B}|\mathbf{R}|$ & \\
\hline \hline C1 & 0.000000 & 295.1700 \\
C2 & 0.000000 & 295.1700 \\
C3 & 0.000000 & 295.1700 \\
C4 & 0.000000 & 295.1700 \\
C5 & 0.000000 & 295.1700 \\
C6 & 1.000000 & 295.1700 \\
C7 & 0.000000 & 295.1700 \\
C8 & 0.000000 & 295.1700 \\
C9 & 0.000000 & 295.1700 \\
C10 & 0.000000 & 295.1700 \\
C11 & 0.000000 & 295.1700 \\
C12 & 0.000000 & 295.1700
\end{tabular}

Figura 9. Cálculo del número de operarios Contratados por periodo.

La figura 10, indica, el número de operarios despedidos en cada mes, mostrado en la columna Value con sus respectivos costos a reducir. $\$ 210.47$

\begin{tabular}{rll}
\hline $\mathbf{Q}] \mathbf{B}|\mathbf{B}| \boldsymbol{\beta}|\boldsymbol{R}|$ & \\
\hline D1 & 5.000000 & 210.4700 \\
D2 & 2.000000 & 210.4700 \\
D3 & 0.000000 & 210.4700 \\
D4 & 0.000000 & 210.4700 \\
D5 & 0.000000 & 210.4700 \\
D6 & 0.000000 & 210.4700 \\
D7 & 0.000000 & 210.4700 \\
D8 & 0.000000 & 210.4700 \\
D9 & 1.000000 & 210.4700 \\
D10 & 0.000000 & 210.4700 \\
D11 & 0.000000 & 210.4700 \\
D12 & 3.000000 & 210.4700
\end{tabular}

Figura 10. Cálculo del número de operarios Despedidos por periodo.

La figura 11, expresa, el número de Modelos M4 que deben existir en inventario, el cual se indica en la columna de Value con su respectivo valor para reducir los costos. $\$ 10.88$

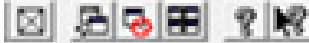

\begin{tabular}{lll}
\hline I1 & 4.000000 & 10.88000 \\
I2 & 4.000000 & 10.88000 \\
I3 & 0.000000 & 10.88000 \\
I4 & 12.00000 & 10.88000 \\
I5 & 2.000000 & 10.88000 \\
I6 & 6.000000 & 10.88000 \\
I7 & 0.000000 & 10.88000 \\
I8 & 22.00000 & 10.88000 \\
I9 & 17.00000 & 10.88000 \\
I10 & 5.000000 & 10.88000 \\
I11 & 0.000000 & 10.88000 \\
I12 & 0.000000 & 10.88000
\end{tabular}

Figura 11. Cálculo del inventario Modelo M4 por periodo

La figura 12, muestra, los productos que pueden quedar sin entregar en cada mes, cuyo valor se muestra en la columna de Value, y el costo por inventario agotado es igual a $\$ 103.26$ por mes.

\begin{tabular}{|c|c|c|}
\hline F1 & 0.000000 & 103.2600 \\
\hline F2 & 0.000000 & 103.2600 \\
\hline E3 & 1.000000 & 103.2600 \\
\hline E4 & 0.000000 & 103.2600 \\
\hline E5 & 0.000000 & 103.2600 \\
\hline F6 & 0.000000 & 103.2600 \\
\hline 27 & 0.000000 & 103.2600 \\
\hline F8 & 0.000000 & 103.2600 \\
\hline F9 & 0.000000 & 103.2600 \\
\hline F10 & 0.000000 & 103.2600 \\
\hline F11 & 0.000000 & 103.2600 \\
\hline F12 & 0.000000 & 103.2600 \\
\hline
\end{tabular}

Figura 12. Cálculo del inventario agotado Modelo M4 por periodo.

La figura 13, muestra la producción que debería darse para satisfacer cada una de las restricciones ya planteadas, y así el costo de producción sea el óptimo. Columna Value.

\begin{tabular}{|c|c|c|}
\hline \multicolumn{3}{|c|}{ Q] } \\
\hline P1 & 150.0000 & 0.000000 \\
\hline P2 & 115.0000 & 0.000000 \\
\hline P3 & 115.0000 & 0.000000 \\
\hline P4 & 136.0000 & 0.000000 \\
\hline PS & 138.0000 & 0.000000 \\
\hline P6 & 120.0000 & 0.000000 \\
\hline P7 & 144.0000 & 0.000000 \\
\hline P8 & 144.0000 & 0.000000 \\
\hline P9 & 115.0000 & 0.000000 \\
\hline P10 & 138.0000 & 0.000000 \\
\hline P11 & 115.0000 & 0.000000 \\
\hline P12 & 120.0000 & 0.000000 \\
\hline
\end{tabular}

Figura 13. Cálculo del inventario agotado Modelo M4 por periodo.

Para este modelo se muestra la tabla 7, que indica los costos de producción por mes para el año 2020.

\begin{tabular}{|c|c|c|c|c|c|c|c|}
\hline \begin{tabular}{|c|} 
Características \\
Meses
\end{tabular} & 1 & 2 & $\ldots$ & $\ldots$ & $\ldots$ & 11 & 12 \\
\hline $\begin{array}{c}\text { Costo de un } \\
\text { trabajador en el } \\
\text { mes (Wt) }\end{array}$ & $\begin{array}{c}\$ \\
699,84\end{array}$ & $\begin{array}{c}\$ \\
699,84\end{array}$ & $\begin{array}{c}\$ \\
699,84\end{array}$ & $\begin{array}{c}\$ \\
699,84\end{array}$ & $\begin{array}{c}\$ \\
699,84\end{array}$ & $\begin{array}{c}\$ \\
699,84\end{array}$ & $\begin{array}{c}\$ \\
699,84\end{array}$ \\
\hline $\begin{array}{c}\text { Costo de } \\
\text { contratación y } \\
\text { capacitación }(\mathrm{Ct})\end{array}$ & $\begin{array}{c}\$ \\
295,17\end{array}$ & $\begin{array}{c}\$ \\
295,17\end{array}$ & $\begin{array}{c}\$ \\
295,17\end{array}$ & $\begin{array}{c}\$ \\
295,17\end{array}$ & $\begin{array}{c}\$ \\
295,17\end{array}$ & $\begin{array}{c}\$ \\
295,17\end{array}$ & $\begin{array}{c}\$ \\
295,17\end{array}$ \\
\hline \begin{tabular}{|c|} 
Costo de despido \\
(Dt)
\end{tabular} & $\begin{array}{c}\$ \\
210,47\end{array}$ & $\begin{array}{c}\$ \\
210,47\end{array}$ & $\begin{array}{c}\$ \\
210,47\end{array}$ & $\begin{array}{c}\$ \\
210,47\end{array}$ & $\begin{array}{c}\$ \\
210,47\end{array}$ & $\begin{array}{c}\$ \\
210,47\end{array}$ & $\begin{array}{c}\$ \\
210,47\end{array}$ \\
\hline \begin{tabular}{|c|} 
Costo de \\
mantenimiento del \\
inventario (It)
\end{tabular} & $\$ 10,88$ & $\$ 10,88$ & $\$ 10,88$ & $\$ 10,88$ & $\$ 10,88$ & $\$ 10,88$ & $\$ 10,88$ \\
\hline $\begin{array}{l}\text { Costo marginal } \\
\text { del inventario } \\
\text { agotado }(\mathrm{Ft})\end{array}$ & $\$ 13,26$ & $\$ 13,26$ & $\$ 13,26$ & $\$ 13,26$ & $\$ 13,26$ & $\$ 13,26$ & $\$ 13,26$ \\
\hline
\end{tabular}

Tabla 7. Cálculo del inventario agotado Modelo M4 por periodo. 


\begin{tabular}{cll}
\hline No & \multicolumn{1}{c}{ Restricción } & \multicolumn{1}{c}{ Costo } \\
\hline$I$ & (Wt) Costo de un trabajador al mes & $\$ 699,84 /$ trabajador \\
\hline 2 & (Ct) Costo de contratación y capacitación & $\$ 295,17$ /trabajador \\
\hline 3 & (Dt) Costo de despido & $\$ 210,47$ /trabajador \\
\hline 4 & (It) Costo de mantenimiento del inventario & $\$ 10,88 /$ modelo \\
\hline 5 & (Ft) Costo marginal del inventario agotado & $\$ 103,26 /$ modelo
\end{tabular}

Tabla 8. Costos de producción en ensamble Modelo M4.

Reemplazando en la ecuación cinco (5) los valores de Costo de la tabla 8 , se obtiene el Modelo Matemático de Programación Lineal para minimizar los costos de producción anual del Modelo M4 $(21,22)$.

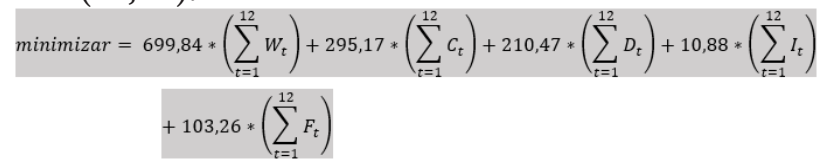

\section{- Resumen de los Resultados para el proceso de} ensamble del Modelo M4

Una vez mostrada la respuesta del programa lingo 19 , se procede a realizar un resumen de esta en la Tabla 9, la cual muestra la producción, número de trabajadores, contratación, despidos, inventario existente e inventario agotado por mes, para que el costo total de este plan para el año 2019 sea de $\$ 198.052,50$.

\begin{tabular}{ccccccc}
\hline Mes & Producción & Trabajadores & Contratación & Despido & Inventario & Faltantes \\
\hline Enero & 150 & 25 & 0 & 5 & 4 & 0 \\
\hline Febrero & 115 & 23 & 0 & 2 & 4 & 0 \\
\hline Marzo & 115 & 23 & 0 & 0 & 0 & 1 \\
\hline Abril & 136 & 23 & 0 & 0 & 12 & 0 \\
\hline Mayo & 138 & 23 & 0 & 0 & 2 & 0 \\
\hline Junio & 120 & 24 & 1 & 0 & 6 & 0 \\
\hline Julio & 144 & 24 & 0 & 0 & 0 & 0 \\
\hline Agosto & 144 & 24 & 0 & 0 & 22 & 0 \\
\hline Septiembre & 115 & 23 & 0 & 1 & 17 & 0 \\
\hline Octubre & 138 & 23 & 0 & 0 & 5 & 0 \\
\hline Noviembre & 115 & 23 & 0 & 0 & 0 & 0 \\
\hline Diciembre & 120 & 20 & 0 & 3 & 0 & 0 \\
\hline Costo total & & & $198.052,50$ & & &
\end{tabular}

Tabla 9. Datos de producción proceso de ensamble del Modelo M4, 2019.

\section{DISCUSION}

Revisando la bibliografía, se registra estudios sobre modelos estadísticos y matemáticos aplicados en el área de textil (cuero), forestal y alimenticio (24).

Según (25), en la investigación que tuvo como propósito modelar el raleo en plantaciones de Pinus caribaea utilizando la programación lineal como herramienta matemática y considerando como restricciones económicas el presupuesto y el combustible asignado a la empresa. Para ello se trabajó con datos obtenidos del Proyecto de Ordenación de la Unidad Silvícola San Juan y Martínez, perteneciente a la Empresa Agroforestal Pinar del Río - Cuba. La muestra se seleccionó a partir de los rodales que tenían manejo recomendado. El modelo matemático obtenido planifica los raleos en un total de 30 meses con las cantidades óptimas de combustible y presupuesto a emplear para ello, de modo que se maximice el número de rodales a ralear en la unidad silvícola. Considerando los resultados del modelo, la actividad se realizaría en dos años y seis meses, con un consumo total de 973,2 litros de combustible y \$ 298708,74 de presupuesto. Además, ordena los rodales que debe recibir el tratamiento silvicultural, mostrando el tipo de raleo y el orden de este, de modo que se optimice la actividad forestal (25).

Según (23), en su trabajo que estudió la cadena de suministro tomando como referencia el sector productivo de neumáticos en Ecuador, mediante la utilización de un modelo de Programación Lineal Entera Mixta. Concluyeron que: para el diseño de un modelo estadístico para la cadena de suministro; fue trascendental la determinación de la cantidad de materias primas que deben ser entregadas, así como que proveedores deben suministrar a la planta, basados en la demanda de cada producto. Adicionalmente se debió tomar en cuenta que para el diseño de un modelo se deberá plantear diferentes escenarios; en un escenario optimista se sugiere la ampliación de las zonas de consumo debido a que varios centros de distribución convergen en algunos sectores, el modelo conservador indica que los centros de distribución son óptimos, mientras que el escenario pesimista requiere la instalación de más centros de distribución. Por otra parte, se realiza el diseño de la red, en donde se puede observar la aplicación del enfoque de Distribuidor Único, ya que cada centro de distribución cubre determinadas zonas, de esta manera se consigue cubrir la demanda de la empresa en todas las zonas de consumo, evitando duplicidad de esfuerzos y recursos. El resultado final del estudio puede ser descrito como el desarrollo de un modelo matemático que permite identificar la mejor composición de la red logística, analizando diferentes escenarios, por lo que estos modelos pueden ser 
adaptados a diferentes segmentos de la industria (23).

Como podemos observar en los resultados expuestos en los puntos anteriores y comparando con investigaciones similares, las empresas han logrado reducciones significativas en los costos de producción, los inventarios, costos de compra, costos de calidad y lead time aplicando modelos matemáticos, como es el caso de investigación con el tema "Modelo matemático para la planificación de la producción del sector cuero en la parroquia de Quisapincha” (5).

En esta investigación presentamos un modelo matemático para minimizar los costos de los parámetros inmersos en la producción como son; costo de un trabajador por mes, costo de contratar y capacitar un trabajador, costo de despedir un trabajador, costo de mantener el inventario, $y$ costo del inventario agotado.

Con el Modelo propuesto se logra optimizar el talento Humano en función del número de unidades planificadas a producir por mes para satisfacer la demanda del mercado, con un ahorro de \$ 699,84 por trabajador, minimizando los costos de contratar y despedir un trabajador, reduciendo de esta manera los costos de producción mensual, frente al esquema anterior estos costos no se visualizaban, manteniendo el mismo número de trabajadores para producciones altas y bajas. Optimizando los recursos, con la capacidad instalada de la planta, aplicando el software matemático Lingo 19, para el modelo matemático propuesto, ahora podemos conocer el costo de producción total anual que es de \$198.052,50.

\section{CONCLUSIONES}

El diseño del Modelo Matemático se inicia considerando un número de treinta (30) operarios, por lo que entre las ventajas del modelo y en base al plan de producción, el programa Lingo 19, determina si se contrata o se despide trabajadores, teniendo en cuenta el inventario existente y el agotamiento de inventario, de este modo, podemos saber cuál sería el costo para una determinada producción optimizando los recursos al máximo, lo que anteriormente no lo sabíamos.

El modelo matemático nos permite determina el número de trabajadores en función de la producción, optimizando de esta manera este recurso, es decir para una producción mensual de 144 unidades del Modelo M4, se requiere de 24 trabajadores, con un ahorro de $\$ 699,84$ por trabajador al mes. Si bien es cierto, como podemos observar, una limitación de este modelo es estar diseñado para minimizar los costos para una producción de un año.

Para este caso en particular de la empresa Ciauto Cia Ltda., con una producción de seis (6) unidades del Modelo M4 por día, el costo de producción total al año es de $\$ 198.052,50$. Para ello, aplicando el software Estadístico Lingo 19 se determinó las constantes del Modelo Matemático de Programación Lineal para minimizar los costos de producción del Modelo M4. (Función Objetivo).

$\mathrm{R}$ eferencias

1. Aguado, M. Modelo de mejora eficiente y sostenible en un sistema de producción ajustado través de procesos de innovación ambiental. vol 2. 33era ed. Madrid: UNED; 2015

2. Benavides-Pazmiño M. Aplicación del modelo econométrico de SOLOW para el diagnóstico de la productividad por el uso de la tecnología en las PYMES del sector textil del Ecuador [tesis]. Quito: EPN; 2016. Disponible en: http://bibdigital.epn.edu.ec/bitstream/15000/16578/1/CD-7235.pdf

3. Álvarez R. Metodología de la investigación: Operacionalización de Variables. Vol 2. 2da. ed. Medellín: McGraw-Hill;2012.

4. Camino M, Estimación de una función de producción y análisis de la productividad: el sector de innovación global en mercados locales. Estudios Gerenciales [Internet]. 2017 [citado 10 febrero 2021]; 33 (145). 400-411. Disponible en: doi:https://doi.org/10.1016/j.estger.2017.10.004

5. Ortiz-Zurita J. Modelo matemático para la planificación de la producción del sector Cuero en la parroquia de Quisapincha [tesis]. Ambato: UTA; 2017.

6. Huang, Z., Kim, J., Sadri, A., Dowey, S., Dargusch, M. Industry 4.0: Development of a multi-agent system for dynamic value stream mapping in SMEs. Journal of Manufacturing Systems [Internet]. 2019 [citado 03 marzo 2021]; 52: 1-12. Disponible en: doi:https://doi.org/10.1016/j.jmsy.2019.05.001

7. Escobar S. Modelo de estimación estadística «Programa Inclusión Productiva» MIPRO-Ecuador. 
Retos. Revista de Ciencias de la Administración y Economía [Internet]. 2019 [citado 10 marzo 2021]; 9: 303-325. Disponible en: doi:https://doi.org/ 10.17163/ret.n18.2019.08

8. Garcia, E. Herramientas de la planificación de la producción empresarial. Vol 2. 2da ed. Lima: Gestiopolis; 2011.

9. Espinosa C, Muyulema J, Sánchez R, Usca R. Los retos sostenibilistas de las Pymes textileras de la provincia de Tungurahua - Ecuador. Revista Internacional de Investigación e Innovación Tecnológica - RIIIT. 2019; 1: 1-19.

10. González R, Moreno M. Procedimiento para implementación de un sistema de gestión de costos de calidad. Ciencias Holguín. 2015; 4: 1-17.

11. Zotelo R, Mula J, Díaz M, González G. Plan maestro de producción basado en programación lineal entera para una empresa de productos químicos. Revista de Métodos Cuantitativos para la Economía y la Empresa[Internet]. 2017 [citado 12 marzo 2021]; 24: 147-168. Disponible en: doi:http://hdl.handle. net/10419/195385

12. Jaegler Y, Burlat P, Lamouri S, Trentesaux D. The ConWip production control system: a systematic review and classification. Revista Internacional de Investigación de Producción. [Internet]. 2018 [citado 04 marzo 2021]; 17: 5736-5757. Disponible en: doi:https://doi.org/10.1080/00207543.2017.1380325

13. Rodríguez L, Loyo J, López M, González J. Simulación dinámica de un sistema de producción retroalimentado Ingeniería Industrial. Ingeniería Industrial. 2019; 2: 171-182.

14. Vargas J, Muratalla G, Jiménez M. Sistemas de producción competitivos mediante la implementación dela herramienta lean manufacturing. Ciencias Administrativas. 2018 1: 1-21.

15. Sablón N, Orozco E, Lomas C, Montero Y. Plan maestro de producción de una empresa textil. Caso de estudio de Imbabura, Ecuador. EPISTEME. Revista digital de Ciencia, Tecnología e Innovación. 2018; 4: 448-462.

16. Zamora J, Eguía A. Modelo de Gestión Empresarial Balanced Scorecard. TECTZAPIC. Revista de divulgación científica y tecnológica. 2015; 1; 94-98.

17. Gunaki T, Siddiqui F. A Review Paper on Productivity Improvement by Value Stream Mapping. Journal of Emerging Technologies and Innovative. Research. JETIR. 2015; 2: 1119-1124.

18. Gutiérrez H. Control Estadístico de Calidad y Seis Sigma. Vol 1. 3era. ed. México D. F: McGraw-Hill; 2013

19. Karam A, Liviu M, Veres C, Radu H. The contribution of lean manufacturing tools to changeover time decrease in the pharmaceutical industry. A SMED projec. Procedia Manufacturing. [Internet]. 2018 [citado 09 marzo 2021]; 22: 886-892. Disponible en: doi:https://doi.org/10.1016/j.promfg.2018.03.125

20. Boiteux O, Forradella R, Palma R, Guiñazu H. Modelo Matemático Para la Planificación Agregada de la producción de Impsa. IJIE [Internet]. 2010 [citado 04 febrero 2021]; 2: 90-112. Disponible en: http://incubadora.periodicos.ufsc.br/index.php/IJIE/article/viewFile/622/pdf_93

21. Montgomery. Diseño y analisis de experimentos. Vol 2. 2da. ed. Arizona: Limusa Wiley; 2004

22. Bautista J, Batet L, Mateo M. Minimización del coste de almacenaje de combustible nuclear gastado en cápsulas MPC-32 con programación lineal entera mixta: Caso de carga en una etapa.[tesis doctoral]. México: UPC; 2020.

23. Campoverde A, Romero A, Naula B, Loyola M, Coronel T. (2019). Aplicación de un modelo matemático para el diseño de la cadena de suministro en el sector de neumáticos en Ecuador. Espacios [Internet]. 2019 [citado 13 marzo 2021]; 13: 10-21.

24. Hernández R, Fernández C, Baptista M. Metodología de la Investigación. Vol 2. 6ta ed. México D.F. : McGraw-Hill; 2014

25. Castillo B, Aguirre Z. Modelación del raleo mediante el uso de la programación lineal en plantaciones de Pinus caribaea Morelet de la Empresa Agroforestal Pinar del Río, Cuba. Arnaldoa [Internet]. 2018 [citado 16 febrero 2021]; 2: 597-614. Disponible en: doi:http://doi.org/10.22497/arnaldoa.252.25215 